\title{
Optical forces near a nanoantenna
}

\author{
Martin Ploschner, ${ }^{a}$ Michael Mazilu, ${ }^{b}$ Thomas F. Krauss ${ }^{c}$ and Kishan \\ Dholakia $^{d}$ \\ a,b,c,d SUPA, School of Physics and Astronomy, University of St Andrews \\ St Andrews, Fife, KY16 9SS, UK \\ amp399@st-andrews.ac.uk
}

\begin{abstract}
The Maxwell stress tensor method is used to calculate the optical forces acting upon a glass nanosphere in the proximity of gold nanoantenna structures in normal and total internal reflection case. The angle dependance of optical forces over a full range of angles leads to a conclusion that total internal reflection incidence does not bring any particular advantage to trapping efficiency. Multiple trapping sites are found with similar trapping properties for the normal and the total internal reflection case, respectively. The issue of convective heating, which might oppose the optical forces and optical trapping in particular, is also discussed.
\end{abstract}

Keywords: Mie,force,nanoantenna,Maxwell stress tensor.

\section{INTRODUCTION}

The optical confinement of microscopic and nanoscopic objects is a rapidly developing field of optical trapping with many applications in physics, biology and chemistry [1]. In general optical trapping occurs due to the interplay between the gradient and scattering forces acting upon a transparent object. Typically, the gradient force causes objects of higher refractive index than the surrounding media to localise themselves in the brightest parts of the beam. In recent years several methods have been put forward by which one can create high optical gradients particularly close to a surface and thus perhaps create discrete localised enhanced fields suitable as trap sites for nanoparticles in particular. Various such methods in near field trapping have been reported [2]. The field enhancement can also be generated by surface resonators [3] or through the use of microfabricated structures such as slot waveguides [4]. In this area, some of the popular methods, that have been developed over recent years, have a common factor the use of plasmonic enhancement in the proximity of a metallic nanostructure [5]. However, the properties of localized plasmons are highly dependent on the effective refractive index of the medium around the plasmonic structure and so the trapped object can easily influence the plasmon resonance. This brings a new dimension to the physics involved in optical trapping as we have to carefully consider the object-object interaction. In the case of conventional optical tweezers, the scattering problem which leads to optical forces was solved and explored in significant detail [6], [7], [8]. However, very little work has been performed in this regard in the case of plasmonic nanostructures [9]. Altough scattering problems have been solved for many plasmonic structures, the trapped object was not explicitly included in the scattering problem. However, the object-object interaction requires that both objects, the one being trapped and the one used for trapping, have to be included in the formulation of the scattering problem, because otherwise the whole realm of important details for the forces is neglected. It is therefore imperative to include the trapped object in the scattering problem explicitly. In this paper, we explore in detail the nanoantenna case used for trapping of glass nanospheres in normal and total internal reflection incidence cases, which was recently reported [10] and appears to be a promising method of enhanced near field optical trapping. The first part of the paper describes the theoretical basis for calculation of optical forces, the second part of the paper then contains the results of the simulations and the comparison of several experimental situations.

Send correspondence to Martin Ploschner. 


\section{THEORY OF OPTICAL FORCES}

\subsection{Maxwell stress tensor method}

The force exerted on an object positioned in a time-harmonic electromagnetic field of frequency $\omega$ can be obtained by using the law of conservation of linear momentum. The linear momentum is present in the system as either field or mechanical momentum. The sum of these two parts, which is the total momentum of the system, is conserved. In an optical interaction the momentum is transferred from one form to the other. This change of momentum, from field to mechanical, exhibits itself as a mechanical force acting on the object. Indeed, the force $\mathbf{F}$ acting on the object corresponds to the variation of the mechanical momentum $\mathbf{p}$ leading to $\mathbf{F}=\mathrm{d} \mathbf{p} / \mathrm{d} t$. The electromagnetic field momentum flux in the linear medium of relative permittivity $\epsilon_{m}$ and permeability $\mu_{m}$ is represented mathematically by the Maxwell stress tensor

$$
\sigma_{i j}=\epsilon_{0} \epsilon_{m} E_{i} E_{j}+\mu_{0} \mu_{m} H_{i} H_{j}-\frac{1}{2} \delta_{i j}\left(\epsilon_{m} \epsilon_{0} E_{k} E_{k}+\mu_{m} \mu_{0} H_{k} H_{k}\right)
$$

where we used implicit summation on repeating indices and where $E_{i}$ and $H_{i}$ correspond to the electric and magnetic fields. This form of the Maxwell stress tensor can be cast into a simpler form in harmonic fields. Since

$$
E_{i}(\mathbf{r}, t)=\Re\left[E_{i}(\mathbf{r}) e^{i \omega t}\right]=\frac{1}{2}\left(E_{i}(\mathbf{r}) e^{i \omega t}+E_{i}^{*}(\mathbf{r}) e^{-i \omega t}\right),
$$

where $\Re$ is the symbol for real part and $\mathbf{r}$ is the position vector. Using the above equation, we can write the product of any two field components as

$$
\begin{aligned}
E_{i} H_{j} & =\frac{1}{4}\left(E_{i}(\mathbf{r}) e^{i \omega t}+E_{i}^{*}(\mathbf{r}) e^{-i \omega t}\right)\left(H_{j}(\mathbf{r}) e^{i \omega t}+H_{j}^{*}(\mathbf{r}) e^{-i \omega t}\right) \\
& =\frac{1}{2} \Re\left(E_{i} H_{j}^{*}+E_{i} H_{j} e^{2 i \omega t}\right)
\end{aligned}
$$

and because we are only interested in quantities averaged over one optical cycle we can write the cycle averaged Maxwell stress tensor as

$$
\left\langle\sigma_{i j}\right\rangle=\frac{1}{2} \Re\left[\epsilon_{0} \epsilon_{m} E_{i} E_{j}^{*}+\mu_{0} \mu_{m} H_{i} H_{j}^{*}-\frac{1}{2} \delta_{i j}\left(\epsilon_{m} \epsilon_{0} E_{k} E_{k}^{*}+\mu_{m} \mu_{0} H_{k} H_{k}^{*}\right)\right],
$$

where $\langle\ldots\rangle$ corresponds to $\frac{1}{T} \int_{0}^{T} \ldots \mathrm{d} t$ with $T=\frac{2 \pi}{\omega}$. Now imagine an object in volume $V$ surrounded by a closed surface $S$. Since the momentum must be conserved inside the volume $V$ then the non-zero flux flow of electromagnetic momentum across the closed surface $S$ can be explained only by transformation of momentum from electromagnetic to mechanical. The electromagnetic force acting on an object can thus be written as

$$
\left\langle F_{i}\right\rangle=\int_{S}\left\langle\sigma_{i j}\right\rangle n_{j} \mathrm{~d} S
$$

where $n_{j}$ is the outward unit normal to the closed surface $S$.

\subsection{Model assumptions}

In order to determine the optical forces, the fields $E_{i}$ and $H_{i}$ have to be found, since those fields need to be incorporated into the Maxwell stress tensor and subsequently into force field calculations. The problem of force calculation thus reduces to solving the electromagnetic scattering problem. To solve the Maxwell equations, we first need to know the optical properties $\epsilon(\omega)$ and $\mu(\omega)$ of the object. This is by no means trivial, because the optical properties are usually 
measured for a thin layer of material and thus correspond to its bulk properties. The response of the electrons to the external field can be however strongly influenced by the proximity of an interface or surface [11], where the electrons can scatter. If the size of the object reaches the mean free path of the electron, then this scattering significantly influences the movement of the electrons and thus the optical properties of the object. The $\epsilon(\omega)$ and $\mu(\omega)$ must then be corrected to account for this [12]. We chose the geometry of our problem to minimize such a deviation from bulk material measurements. This allowed us to use the Johnson and Christy data for permittivity of gold [13].

The optical properties of the materials involved in the scattering problem allows us to solve the Maxwell's equations using a variaety of methods. In all the subsequent discussions, we will assume that the materials involved are homogeneous and isotropic, which means that no tensorial description for optical properties is needed. Further, linear constitutive relations

$$
\mathbf{j}=\sigma \mathbf{E}, \quad \mathbf{D}=\epsilon \mathbf{E}, \quad \mathbf{B}=\mu \mathbf{H}
$$

are assumed, which is a reasonable assumption for incident intensities used in this paper. Further support for this treatment is given by the fact, that the field enhancement introduced due to resonance of nanostructures is confined to areas outside the metal, which means that it cannot trigger the nonlinear response inside the metal. The linear constituve relations also allow us to treat the calculations as the time-harmonic problem. The last advantage of this treatment lies in the fact that the optical forces resulting from different spectral components can be simply added together, which allows to find the optical forces even in the case of broad spectral source, like the supercontinuum laser for example.

The last assumption is related to the restriction on the velocity of the particle upon which the optical forces are being exerted. The particle's velocity must be assumed sufficiently small so that the Doppler shift effects and the Lorentz forces are negligible. In nanoparticle optical tweezers applications, those assumptions are easily satisfied.

Armed with all these assumptions and limitations to our problem we are now fully prepared to proceed to the solution of the scattering problem.

\section{NUMERICAL SIMULATIONS}

We have used the Radio Frequency (RF) module of Comsol Multiphysics software in scattered harmonic field mode to analyse the problem. In all of the following cases, the sphere was positioned at a certain point $(x, y, z)$, the scattering problem was solved and Maxwell stress tensor method used to calculate the forces acting on the sphere at this point. This process was repeated over a grid of coordinates for the sphere positions to obtain the force fields.

The model was verified by comparing the Finite Element Method (FEM) solution with the Mie theory in the simple case of the sphere in infinite medium. The forces obtained from FEM model and Mie were compared over the whole computational window and the maximum observed deviation from exact Mie solution was $3 \%$. The mesh used for this comparison was the same as that used for the final solution of our nanoantenna-sphere scattering problem, however the optical properties of nanoantenna and substrate were equal to optical properties of the medium at this stage. The maximum element sizes of $8 \mathrm{~nm}$ for the integration boundary of the investigated sphere and $14 \mathrm{~nm}$ for nanoantenna boundary were found to be optimal to provide sufficient accuracy. The comparison revealed the necessity to move the Perfectly Matched Layers (PML) at least $\lambda_{\text {incident }} / 2$ from scattering objects. The reason for this is very simple. The scattered field has an evanescent character in the close proximity of scattering objects and because PML does not absorb evanescent fields, those fields are effectively reflected back into the computational domain and change the solution appreciably. However, the evanescent character of the fields is exponentially decaying with the distance from the scatterer, which means 
that positioning the PML sufficiently far away from the scatterer insures much more accurate solution.

For reasons of numerical stability and reliability, we have also decided to limit the grid for the sphere in such a way, that the minimum distance between nanoantenna and the lowest point of the sphere is $10 \mathrm{~nm}$. The resolution of a narrower region would require a very fine mesh and this, in combination with the necessity to move the PML in sufficient distance and fine coordinate grid for the positions of the sphere, would result in a computationally time and memory intensive problem, which was not possible to perform on our computer. We are aware of the fact that this is very inconvenient in terms of force field calculations as the magnitude of the force is exponentially increasing as we approach closer to nanostructure, but in terms of incident wavelength and the size of structures involved, $10 \mathrm{~nm}$ is just a fraction of these values and basically corresponds to the case of sphere touching the nanostructure. Further, simple extrapolation procedure seems to give very accurate predictions on the magnitude of the forces for smaller separations than $10 \mathrm{~nm}$, which was tested in a special case of a sphere in a central trap. An order of magnitude estimate of the optical forces for smaller separations can thus be obtained in this way.

\subsection{The normal incidence}

We first analysed the case of the glass sphere of refractive index $n=1.5$ with a diameter of $100 \mathrm{~nm}$ in the close proximity of antenna resonant at $1064 \mathrm{~nm}$ in water (Fig. 1). Each antenna

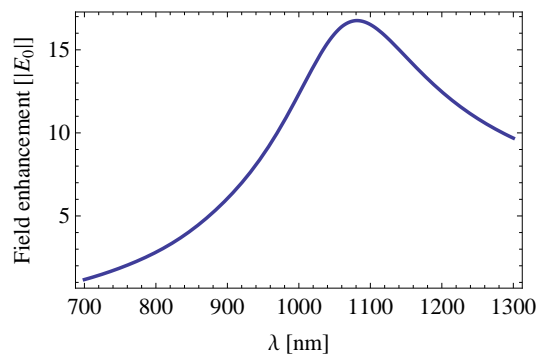

Fig. 1. The field enhancement factor in the middle point of the antenna gap shows resonance at about $1064 \mathrm{~nm}$ in water.

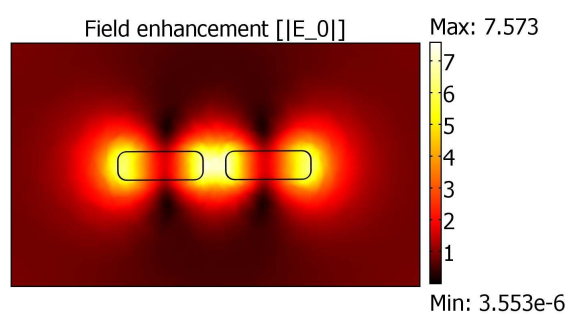

(a)

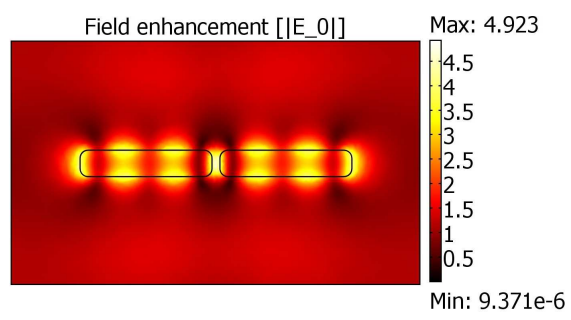

(b)

Fig. 2. (a) Comparison of the field enhancement profile $20 \mathrm{~nm}$ above a) our nanoantenna geometry; b) geometry presented in Ref. [10] for their respective resonant wavelengths of a) $1064 \mathrm{~nm}$ and b) $800 \mathrm{~nm}$ in water.

arm supports $\lambda_{\text {eff }} / 2$ mode with $\lambda_{\text {eff }}=300 \mathrm{~nm}$. The effective mode wavelength is an important parameter for the efficient coupling between two antenna arms and is closely related to the field enhancement in the gap [14]. The corresponding field enhancement for our nanoantenna and 
that used in Ref. [10] is slightly better in our case (Fig. 2). However, the optimal trap nanoantenna structure depends not only on the local enhanced fields in the proximity of the nanoantenna, which might be optimized using comprehensive treatment of the problem in Ref. [14], but also on the overlap of this field with the volume of the particle, which has to be taken into account when designing the structure. Further, the strongest fields in the middle of the gap are not actually fully accessible to the particle, in contrast to the side traps that are more accessible, which might quite generally lead to the similar trapping properties for central and side traps of the nanoantenna structure. The antenna geometry presented in Ref. [10] also supports higher order mode, which results in many hot spots in the proximity of antenna. The separation of the hot spots is not as clear as in our case as well. For these reasons, we have decided to analyze in detail our geometry (Fig. 3). The field used for excitation of localized plasmon in nanoantenna is a plane wave normal incident to the antenna with polarization parallel to the longer axis of antenna. The incident intensity in the computational domain was $17 \mathrm{~mW} / \mu \mathrm{m}^{2}$, which corresponds to the plane wave field amplitude of $E_{0}=2.9141 \times 10^{6} \mathrm{Vm}^{-1}$. The resulting force field

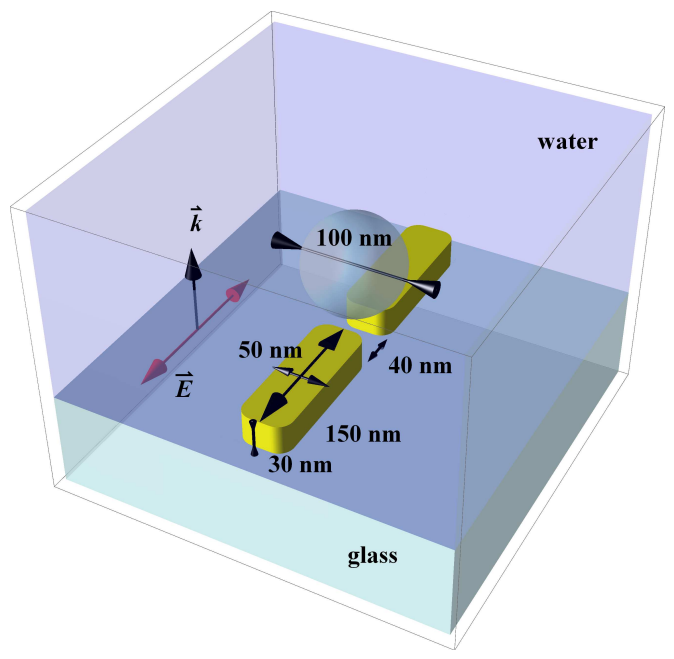

Fig. 3. 3D view of the geometry considered. The two yellow blocks represent the gold nanoantenna on top of the glass and submerged in water. The incident field is in normal incidence and polarization is parallel to the longer axis of antenna. The radius of curvature of the rounding of nanoantenna edges is $15 \mathrm{~nm}$.

is shown on Fig. 4 and 5a,b. There are three traps, one in the center and one at each end of the nanoantenna. The magnitude of optical forces in the central and side traps is very similar and sufficient for optical trapping since the extraction energy in all three traps is several $k T$ 's (see Fig. 6a). The extraction energy profile for a glass sphere along the line parallel to the longer axis of nanoantenna $10 \mathrm{~nm}$ above the structure also confirms the similarity of the central and side traps. This behaviour is further supported when directly comparing the trapping forces for small displacements from the stable trap position (see Fig. $6 \mathrm{~b}$ and 7a,b). This multiple trap site feature might be very inconvenient in terms of trapping, since it seems, that there is no favourable position for trapping. This loss of control over particle position could have serious implications for applications, where immobilization needs to be combined with specific position in the enhanced field around nanostructures as is the case for Surface Enhanced Raman Spectroscopy. 


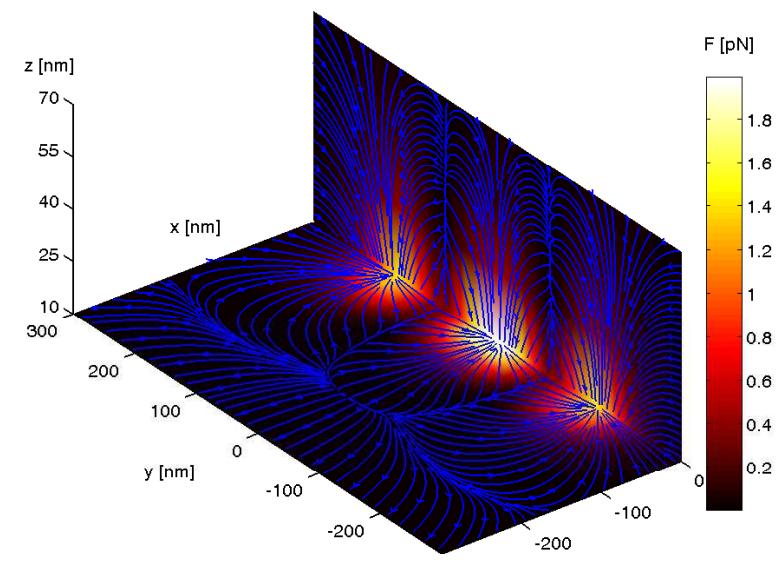

Fig. 4. Streamlines and magnitude of forces acting on the glass particle with its lowest point at distance $z$ from the nanoantenna. Three bright spots correspond to the locations of the traps.

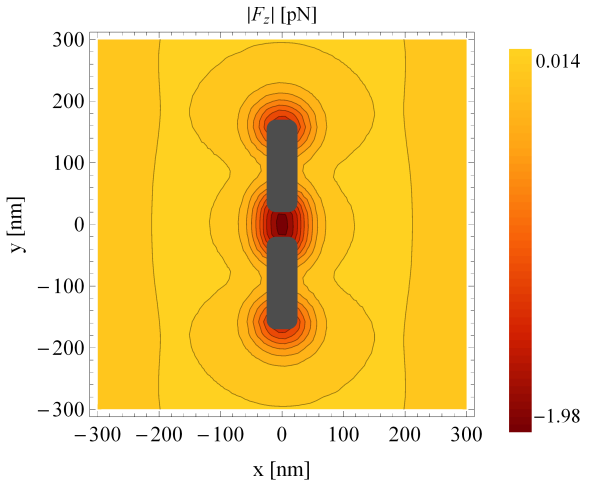

(a)

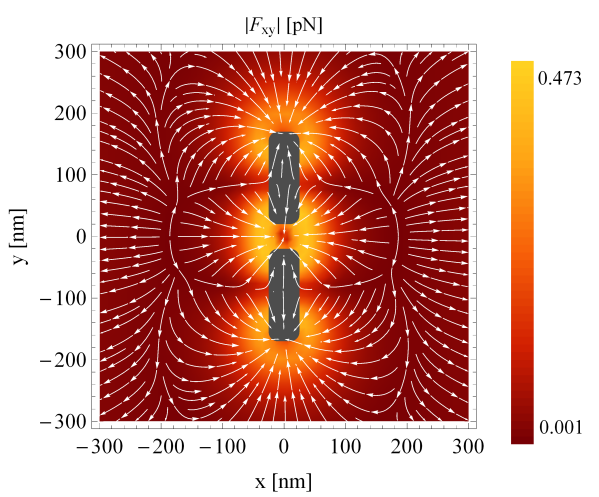

(b)

Fig. 5. (a) The magnitude of $\left|F_{z}\right|$ and (b) $\left|F_{x y}\right|$ with streamlines for the lowest point of sphere $10 \mathrm{~nm}$ from the nanostructure.

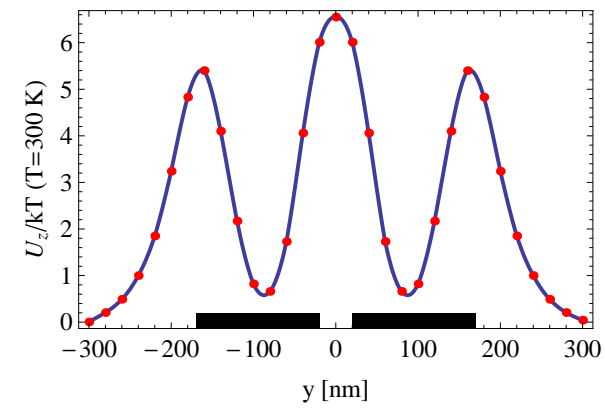

(a)

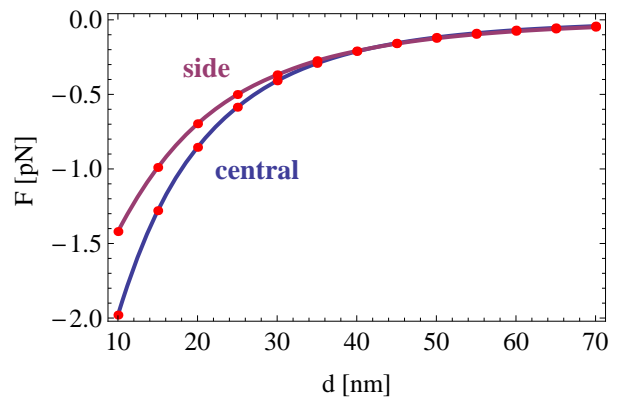

(b)

Fig. 6. (a) The extraction energy needed for a glass particle with its lowest point $10 \mathrm{~nm}$ above nanoantenna to be moved by $60 \mathrm{~nm}$ in the $z$-direction, where the antenna induced forces became negligible. Traps are in the positions of the maxima of the extraction energy profile. (b) Vertical force acting on the sphere in central trap and side traps as a function of the distance of the sphere's lowest point from nanoantenna. The negative sign of the force corresponds to the force pointing down towards the nanoantenna. 


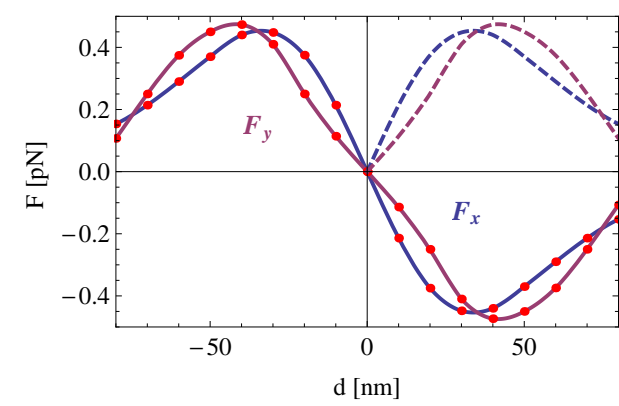

(a)

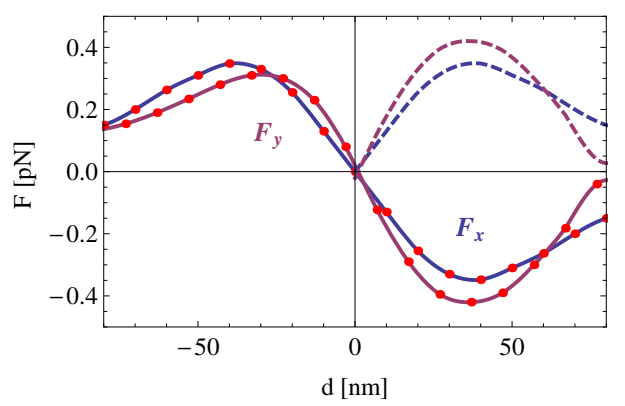

(b)

Fig. 7. Lateral forces acting on a glass sphere with its lowest point $10 \mathrm{~nm}$ above the structure in (a) central and (b) side traps, where $d$ is the displacement from the center of the trap. The dashed line is the reflection of the graph around horizontal axis for $d>0$, which allows easier assessment of the symmetry or asymmetry of the lateral forces.

\subsection{The total internal reflection (TIR)}

We have seen in the previous section, that the central and side traps are very similar. In this section we want to determine the effect of the angle of the incident plane wave on these trapping sites. One case that we study in detail is the total internal reflection at critical angle shown in Fig. 8. This also corresponds to the experimental realization by Quidant et.al. [10]. How-

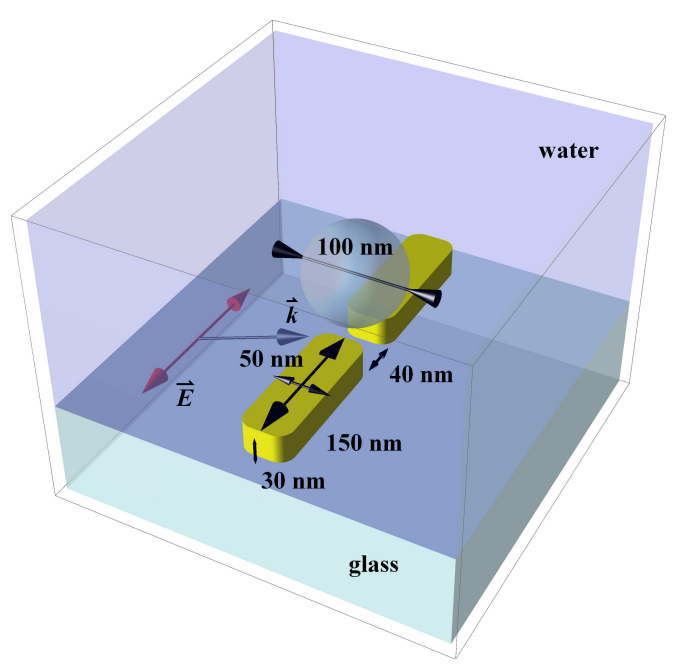

Fig. 8. Total internal reflection incidence, polarization parallel to the longer axis of antenna.

ever, the TIR configuration can be quite cumbersome and complex because specialist optical elements need to be used and it is therefore reasonable to ask whether this approach brings some significant advantages over the case of normal incidence. We first modelled the $z$-forces acting on the particle in central and side traps for the lowest point of sphere $10 \mathrm{~nm}$ above the nanoantenna as a function of incident angle of the plane wave (Fig. 9a). Results clearly show that the optimal angle maximizing the force is the critical angle. The angle dependance of force is closely resembling the intensity dependance on agle at the interface of two semi-infinite media presented in [15], [16]. The similarity can be explained in the following way. The intensity dependance at the interface from [15], which is equivalent to the effective intensity driving the 


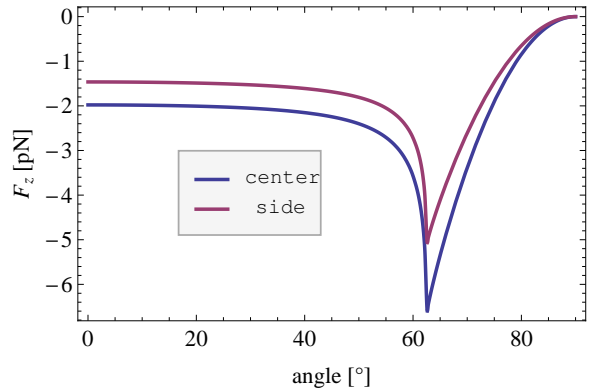

(a)

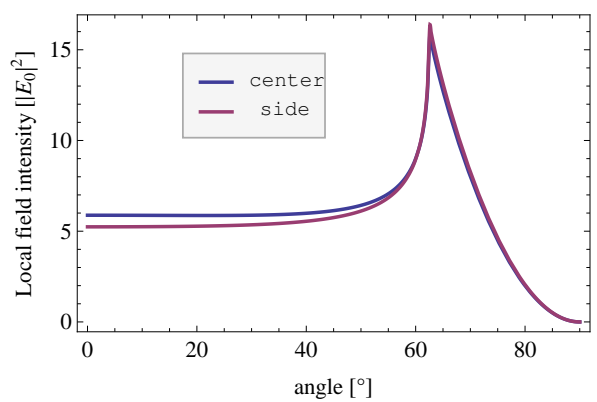

(b)

Fig. 9. (a) The $F_{z}$ force as a function of incident angle in the central and side traps for a lowest point of sphere $10 \mathrm{~nm}$ above the nanoantenna. (b) The local field intensity inside the particle normalized to $E_{0}=2.9141 \times 10^{6} \mathrm{Vm}^{-1}$ averaged over the volume of the particle positioned in central and side traps.

localized plasmons in the nanoantenna, is projected into higher local fields (Fig. 9b) averaged over the particle volume reaching the maximum for critical angle. The increase in the intesity of local fields is then further projected into increased forces acting on the particle reaching the maximum again for the critical angle. However, the undelying principle of all optical trapping experiments is to maximize the trapping force and at the same time minimize the local fields interacting with the particle. Since the damage treshold of the trapped particles or living cells is not affected by global field intensity, but rather by local field intensity, the question arises whether the increase in optical force for TIR incidence is not just equivalent to increase of local field intensity, which can be easily achieved in normal incidence by simply increasing the global intensity of the laser. Fig. $9 \mathrm{~b}$ actually shows that the force $F_{z}$ and the local field intensity (nor-

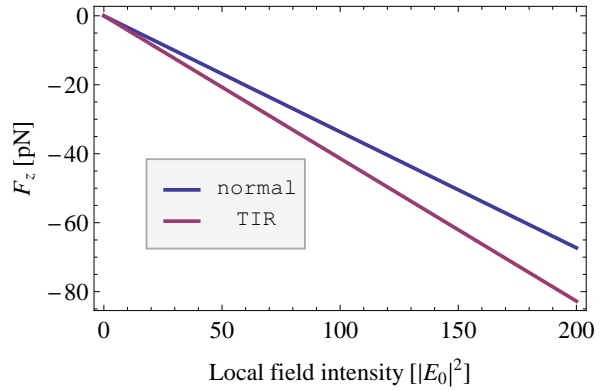

(a)

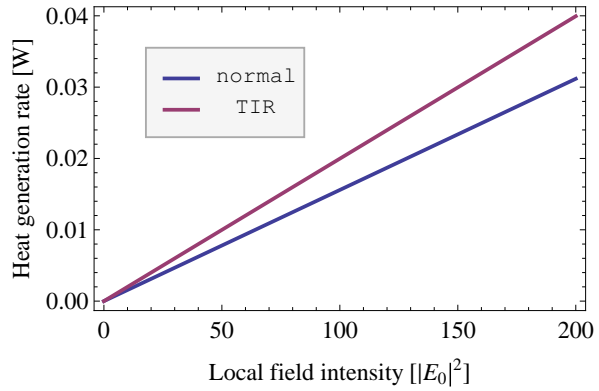

(b)

Fig. 10. (a) The $F_{z}$ force as a function of local field intensity averaged over the volume of the particle shows slight improvement $(23 \%)$ in trapping efficiency for the TIR illumination. (b) The heat generation rate in nanoantenna negatively affecting the thermal dynamics in the proximity of nanoantenna is slightly worse ( $28 \%$ higher) for the case of TIR illumination.

malized to $E_{0}=2.9141 \times 10^{6} \mathrm{Vm}^{-1}$ ) averaged over the volume of the particle are increased by roughly the same factor. Thus, altough the forces might seem to be optimized for critical angle, the same angle gives highest optical fields inside the particle. The better description of the efficiency of the trap is to directly relate local field intensity inside the particle, which drives the strength of light-matter interaction, to forces acting on the particle. We therefore looked at the dependance of force as a function of local field intensity averaged over the volume of the 
particle for the cases of normal incidence and TIR incidence at the critical angle (Fig. 10).

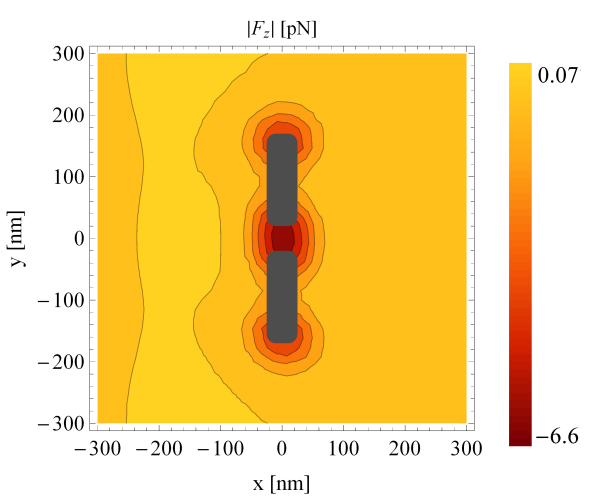

(a)

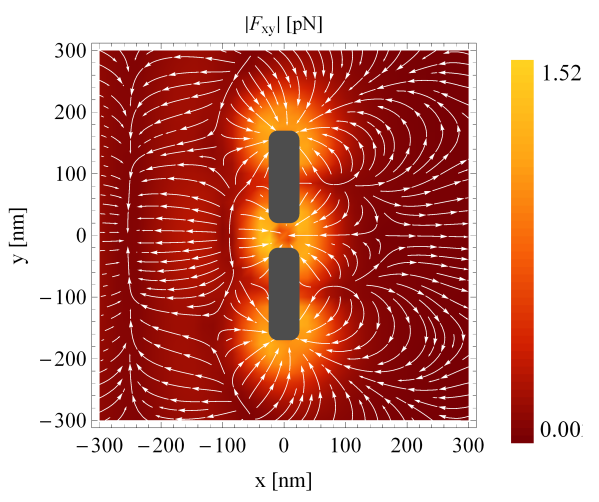

(b)

Fig. 11. (a) The magnitude of $\left|F_{z}\right|$ and (b) $\left|F_{x y}\right|$ with streamlines for the lowest point of sphere $10 \mathrm{~nm}$ from the nanostructure. Notice, that the symmetry of the problem is broken. The incident plane wave is incident at critical angle from the left.

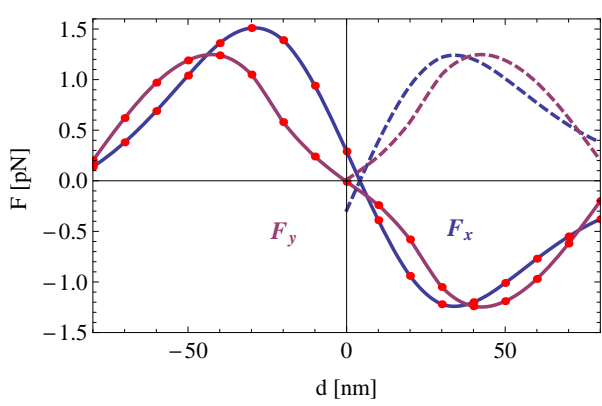

(a)

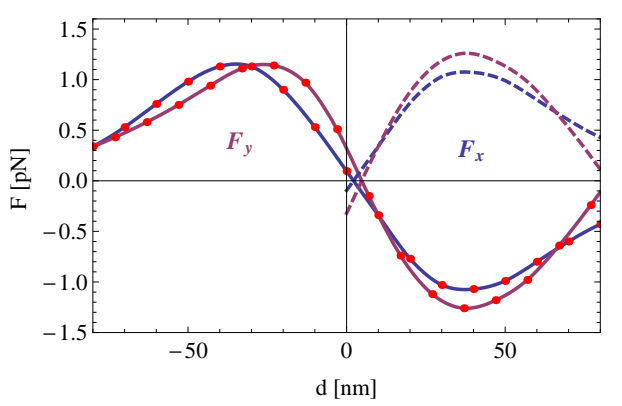

(b)

Fig. 12. Critical angle incidence lateral forces acting on a glass sphere with its lowest point $10 \mathrm{~nm}$ above the structure in (a) central and (b) side traps, where $d$ is the displacement from the center of the trap in normal incidence case. The dashed line is again the reflection of the graph around horizontal axis for $d>0$, which allows easier assessment of the symmetry or asymmetry of the lateral forces.

The improvement in trapping efficiency for TIR illumination compared to the normal incidence determined from the ratio of the slopes in Fig. 10a is $23 \%$. On the other hand, the heat generation rate in nanoantenna is $28 \%$ higher for a given local field intensity in the particle for TIR illumination compared to the normal incidence case (Fig. 10b). The TIR incidence thus does not provide any particular advantage over normal incidence case from the point of view of forces normalized in a way to reflect the efficiency of trapping. To achieve the same magnitude of force, roughly the same local field intensity inside the particles is observed for both illumination cases. The TIR incidence case further provides slightly worse performance in terms of heating, which might induce stronger thermal dynamics, which could completely cancel the $23 \%$ gain in forces as compared to normal incidence.

In addition, the TIR critical angle incidence further introduces assymetries to the trapping sites (Fig. 11 and Fig. 12), which might further decrease the performance of the nanoantenna 


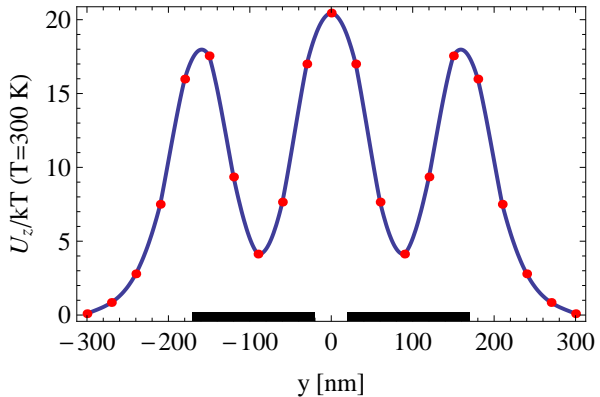

(a)

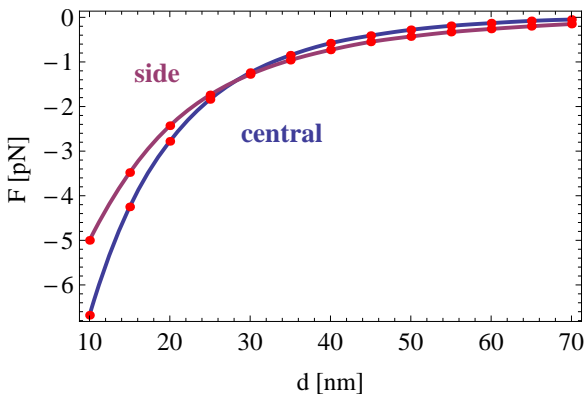

(b)

Fig. 13. (a) The extraction energy needed for a glass particle with its lowest point $10 \mathrm{~nm}$ above nanoantenna to be moved by $60 \mathrm{~nm}$ in the $z$-direction for critical angle incidence. Antenna induced forces became negligible at this point. (b) Vertical force acting on the sphere in central trap and side traps as a function of the distance of the sphere's lowest point from nanoantenna for critical angle incidence.

stucture. As can be seen on Fig. 12, the TIR case significantly breaks the symmetry of lateral forces in central trap, but on the other hand, the symmetry of the side traps is surprisingly improved compared to the case of normal incidence. The extraction energy profile and the $z$-force dependance (Fig. 13) for central and side traps clearly shows the equivalency of central and side traps even for TIR illumination, which further supports our conclusion that no preferred trap position exists near a nanoantenna and that, with this geometry, nanoparticles will be basically randomly trapped in either one of the three similar traps. We can conclude, that TIR does not offer any significant advantages over normal incidence case and the beam delivery system could thus be significantly simplified.

For a comparison of the trapping performance of our nanostructure with respect to the nanostructure presented in Ref. [10], we performed numerical simulation of the extraction energy profile for an experimental situation described in Ref. [10], which means trapping of $200 \mathrm{~nm}$ polystyrene beads at TIR incidence (we assumed critical angle incidence as the angle of incidence was not specified in the paper). To be able to compare the trapping performance the incident intensity used for simulations was again $17 \mathrm{~mW} / \mu \mathrm{m}^{2}$. Since the attractive forces induced by this type of antenna are reversed to repulsive quite close to the antenna structure, we took as a measure of extraction energy the energy needed to move the bead from its initial position to the position, where the force was zero. The extraction energy profile (Fig. 14)

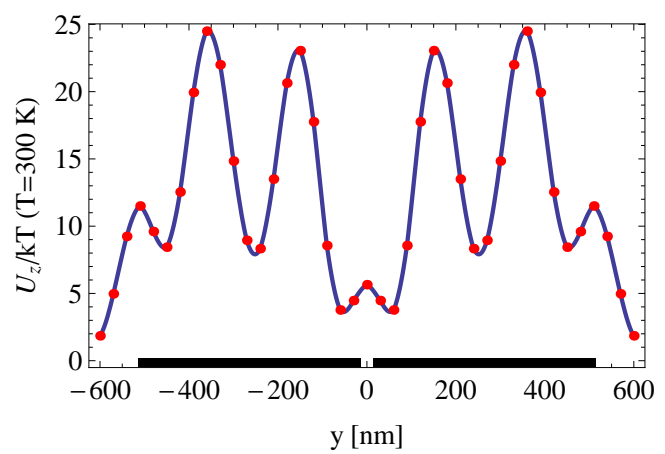

Fig. 14. The extraction energy profile for the geometry presented in Ref. [10] in the case of $200 \mathrm{~nm}$ polystyrene bead. 
clearly shows that the trapping properties in terms of the absolute depth of the potential well are almost the same for both types of antennas. However, the structure supports even more trapping sites (consequence of the higher order mode supported by the nanoantenna), with not as clear boundaries as in the case of our structure. The most efficient trapping appears to be in the second hot spot away from the center of the gap, which is in good agreement with the simulations presented in Ref. [10]. Also notice that the center trap is quite weak, which is a demonstration of the lack of overlap between the enhanced field in the gap and the geometry of the particle. When we further realize that the reference particles are twice as big in Ref. [10] case than in our case and that the gradient forces attracting the particles to the hot spots scale down with the volume of the particles, we can conclude that our structure has similar or even slightly better trapping properties than the structure presented in Ref. [10].

\subsection{Heating issues}

The heating effect in metallic nanostructures is intrinsically related to the amount of incident intensity. As we wish to compare the plasmonic tweezers to conventional optical tweezers, we used $17 \mathrm{~mW} / \mu \mathrm{m}^{2}$ as incident intensity corresponding to a realistic value for conventional tweezing. This incident intensity is also of the same order of magnitude as the one used in Ref. [17] demonstrating plasmonic trapping. Neglecting the thermal effects, the optical force scales linearly with the incident power and as such it is easy to deduce these forces for any illumination power.

From the thermal point of view, an incident power of $17 \mathrm{~mW} / \mu \mathrm{m}^{2}$ corresponds to a heat generation rate of about $1.5 \mathrm{~mW}$ in one antenna arm (at critical angle incidence), which was calculated from time-averaged resistive heating in our model. Taking into account the size of the antenna and the thermal conductivity of the surrounding media, this would roughly imply a local temperature increase to above the boiling point of water. This could lead to bubble formation and very strong hydrodynamic effects disrupting the trapped particle.

This thermal induced effect was also discussed by Quidant et.al. [10] who estimated a temperature change of $1^{\circ} \mathrm{K}$ in the metal. To achieve such a small temperature change the incident intensity had to be decreased to about $40 \mu \mathrm{W} / \mu \mathrm{m}^{2}$ making the absolute trap depth of about orders of magnitude smaller than the one presented in this paper making the trap unstable. Since large scale aggregation of colloid on a surface has also been reported due to the effects of thermophoresis and convection [18] and the same non-optical mechanisms were even used for trapping of $200 \mathrm{~nm}$ polysterene beads [19], we believe that the observed localisation of particles may have been due to means other than optical forces. We shall explore this interplay between optical forces and heating for particles trapped in nanoantennae in future work.

\section{CONCLUSION}

We have performed numerical Maxwell stress tensor calculations to determine the force field near antenna-type nanostructures. Multiple trapping sites were found with very similar trapping properties. This prevents trapping at a unique position, which is a very inconvenient property, since in most of the proposed applications unique position is preferred. Further, the total internal reflection geometry does not show a significant improvement of the trapping properties over normal incidence case, which, from the experimental point of view, could mean significant simplification of the beam delivery system and application of nanoantennae. We have also discussed heating issues, which might significantly disrupt the trapped particle. Decreasing the incident intensity to avoid the heating issues, however, results in very weak optical trapping.

\section{Acknowledgments}

We thank the UK Engineering and Physical Sciences Research Council for funding, KD is a Royal Society-Wolfson Merit Award Holder. 


\section{References}

[1] K. Dholakia, P. Reece, and M. Gu, "Optical micromanipulation,” Chem. Soc. Rev 37, 4255 (2008). [doi:10.1039/b512471a].

[2] K. Dholakia and P. J. Reece, "Near-field optical micromanipulation," in Structured Light and its Applications: An Introduction to Phase-Structured Beams and Nanoscale Optical Forces, D. L. Andrews, Ed., Elsevier Press (2008).

[3] P. J. Reece, V. Garces-Chavez, and K. Dholakia, "Near-field optical micromanipulation with cavity enhanced evanescent waves," Appl. Phys. Lett. 88, 221116 (2006). [doi:10.1063/1.2208272].

[4] A. H. J. Yang, S. D. Moore, B. S. Schmidt, M. Klug, M. Lipson, and D. Erickson, "Optical manipulation of nanoparticles and biomolecules in sub-wavelength slot waveguides," Nature 457, 71-75 (1 January 2009). [doi:10.1038/nature07593].

[5] M. Righini, C. Girard, and R. Quidant, "Light-induced manipulation with surface plasmons," J. Opt. A.: Pure Appl. Opt. 10, 093001 (2008). [doi:10.1088/14644258/10/9/093001].

[6] J. P. Barton, D. R. Alexander, and S. A. Schaub, "Theoretical determination of net radiation force and torque for a spherical particle illuminated by a focused laser beam," J. Appl. Phys. 66(10), 4594-4602 (1989). [doi:10.1063/1.343813].

[7] T. A. Nieminen, V. L. Y. Loke, A. B. Stilgoe, G. Knoner, A. M. Branczyk, N. R. Heckenberg, and H. Rubinsztein-Dunlop, “Optical tweezers computational toolbox," J. Opt. A: Pure Appl. Opt. 9, S196-S203 (2007). [doi:10.1088/1464-4258/9/8/S12].

[8] O. Farsund and B. U. Felderhof, "Force, torque, and absorbed energy for a body of arbitrary shape and constitution in an electromagnetic radiation field," Physica A 227, 108-130 (1996). [doi:10.1016/0378-4371(96)00009-X].

[9] V. Yannopapas, "Optical forces near a plasmonic nanostructure," Phys. Rev. B 78, 045412 (2008). [doi:10.1103/PhysRevB.78.045412].

[10] M. Righini, P. Ghenuche, S. Cherukulappurath, V. Myroshnychenko, F. J. G. de Abajo, and R. Quidant, "Nano-optical trapping of rayleigh particles and escherichia coli bacteria with resonant optical antennas," Nano Lett. 9, 33873391 (2009). [doi:10.1021/nl803677x].

[11] A. Kawabata and R. Kubo, "Electronic properties of fine metallic particles. ii. plasma resonance absortion,” J. Phys. Soc. Jpn 21, 1765-1772 (1966). [doi:10.11143/JPSJ.21.1765].

[12] M. Dienerowitz, M. Mazilu, and K. Dholakia, "Optical manipulation of nanoparticles: a review,” Journal of Nanophotonics 2, 021875 (2008). [doi:10.1117/1.2992045].

[13] P. B. Johnson and R. W. Christy, "Optical constants of the nobel metals," Phys. Rev. B 6(12), 4370 (1972). [doi:10.1103/PhysRevB.6.4370].

[14] H. Fischer and O. J. F. Martin, "Engineering the optical response of plasmonic nanoantennas," Optics Express 16, 9144-9154 (2008). [doi:10.1364/OE.16.009144].

[15] F. de Fornel, Evanescent Waves: From Newtonian Optics To Atomic Optics, Springer (2001).

[16] R. F. Marchington, M. Mazilu, S. Kuriakose, V. Garces-Chavez, P. J. Reece, T. F. Krauss, M. Gu, and K. Dholakia, "Optical deflection and sorting of microparticles in a near-field optical geometry,” Optics Express 16, 3712-3726 (2008). [doi:10.1364/OE.16.003712].

[17] A. N. Grigorenko, N. W. Roberts, M. R. Dickinson, and Y. Zhang, "Nanometric optical tweezers based on nanostructured substrates," Nature Photonics 2, 365-370 (2008). [doi:10.1038/nphoton.2008.78].

[18] V. Garces-Chavez, R. Quidant, P. J. Reece, G. Badenes, L. Torner, and K. Dholakia, “Extended organization of colloidal microparticles by surface plasmon polariton excitation," Phys. Rev. B 73, 085417 (2006). [doi:10.1103/PhysRevB.73.085417].

[19] D. Braun and A. Libchaber, "Trapping of dna by thermophoretic depletion and convection,” Phys. Rev. Lett. 89(18), 188103 (2002). [doi:10.1103/PhysRevLett.89.188103]. 